\title{
BMJ Open Home polygraphic recording with telemedicine monitoring for diagnosis and treatment of sleep apnoea in stroke (HOPES Study): study protocol for a single-blind, randomised controlled trial
}

Stefan Thomas Kotzian, ${ }^{1}$ Angela Schwarzinger, ${ }^{1}$ Sandra Haider, ${ }^{1}$ Bernd Saletu, ${ }^{2}$ Josef Spatt, ${ }^{1}$ Michael Timothy Saletu ${ }^{1}$

To cite: Kotzian ST,

Schwarzinger A, Haider S, et al. Home polygraphic recording with telemedicine monitoring for diagnosis and treatment of sleep apnoea in stroke (HOPES Study): study protocol for a single-blind, randomised controlled trial. BMJ Open 2018;8:e018847. doi:10.1136/ bmjopen-2017-018847

- Prepublication history for this paper is available online. To view these files, please visit the journal online (http://dx.doi. org/10.1136/bmjopen-2017018847).

Received 25 July 2017 Revised 11 September 2017 Accepted 28 September 2017

\section{CrossMark}

${ }^{1}$ Department of Diagnostics, NRZ Rosenhügel Wien, Vienna, Austria

${ }^{2}$ Medical University of Vienna, Vienna, Austria

Correspondence to Stefan Thomas Kotzian; stefan.kotzian@nrz.at

\section{ABSTRACT}

Introduction Meta-analyses report that more than $50 \%$ of patients who had a stroke suffer from moderate to severe sleep apnoea (SA), with adherence rates to positive airway pressure (PAP) therapy of only $30 \%$. The primary objective of this study is to determine whether PAP adherence in patients who had a stroke with obstructive sleep apnoea (OSA) can be improved by a PAP training strategy during inhospital rehabilitation combined with a telemedicine monitoring system after discharge. Further objectives are (1) to compare the validity of a non-attended level-III polygraphy with that of a level-Il polysomnography (PSG) in the diagnosis of SA, (2) to compare the validity of an apnoea-hypopnoea index (AHI) yielded by the PAP device with that obtained during PSG, (3) to determine changes in nocturnal systolic blood pressure (BP) due to PAP therapy with the pulse transit time (PTT) method and (4) to assess the impact of telemonitored PAP therapy on neurorehabilitation outcome parameters. Methods and analyses Single-blind, monocentre, randomised controlled trial. It includes 55 patients who had a subacute stroke, aged 19-70 years, with moderate to severe OSA, who have undergone successful PAP training and titration at the neurorehabilitation unit. Patients are randomised to either a standard care group or a telemedicine group. PAP adherence, sleep and respiratory variables, subjective and objective sleep quality, systolic BP (PTT method) of the two groups are compared after 3 months and 1 year as well as cognitive and motor neurorehabilitation outcome parameters, quality of life and PAP satisfaction. Additionally, intranight AHI/total sleep time versus AHI/time in bed and night-to-night variability of the $\mathrm{AH}$ are assessed. Ethics and dissemination Before screening, all participants will be provided with oral and written information. The study will be disseminated by peer-reviewed publications and conference presentations.

Trial registration number NCT02748681; Pre-results.

\section{INTRODUCTION}

Sleep apnoea and stroke

Sleep apnoea (SA) is characterised by cessation of breathing during sleep and can be caused by an obstruction of the upper airway (obstructive apnoea), loss of ventilatory effort

\section{Strengths and limitations of this study}

- This is the first study using a telemedicine tool to increase positive airway pressure adherence in patients who had a stroke.

- This study adopts a randomised, single-blind, parallel design including female and male patients who had a stroke typically referred to a strokerehabilitation unit.

- Results are limited because of a monocentre design.

- Type-II non-monitored polysomnography (PSG) is used instead of type-I PSG

(central apnoea) or a combination of the two, resulting in oxygen desaturation, sleep fragmentation and acute surges in pulmonary and systemic arterial blood pressure (BP). ${ }^{1}$

Stroke is the third most common cause of death in developed countries worldwide. Each year $\approx 795000$ people experience a new or recurrent stroke (ischaemic or haemorrhagic). ${ }^{2}$

Longitudinal and interventional studies have shown a causal interaction between SA and several conditions of cerebrovascular diseases. $^{13-5}$ The studies available provide sufficient data to establish obstructive sleep apnoea (OSA) as a negative predictor for a 2-3 times higher all-cause mortality and recurrent vascular events following stroke or a transitory ischaemic attack. ${ }^{6-8}$

According to a recent meta-analysis, the percentage of patients with relevant OSA is higher in stroke units $(64 \%)$ and rehabilitation units $(57 \%)$ than in stroke cohort studies $(45 \%) .^{9}$ Polygraphic inhospital sleep studies at the NRZ Rosenhügel confirmed a high prevalence of moderate and severe SA in $56 \%$ of patients who had a stroke enrolled for rehabilitation. ${ }^{10}$ Screening questionnaires 
and/or clinical interviews demonstrated low diagnostic utility. ${ }^{10} \mathrm{SA}$ is also linked to poor functional status in the subacute phase. ${ }^{11}$

Although the prevalence of SA in patients who had a stroke is very high, sleep studies are not routinely performed in stroke rehabilitation units. And for several reasons (eg, waiting time of 6-12 months in Austria, disability and immobility caused by stroke), access to sleep laboratories for patients who had a stroke is limited.

\section{Diagnosing SA in patients who had a stroke}

Current guidelines recommend one night of attended polysomnography (PSG) for the diagnosis of OSA and one separate positive airway pressure (PAP) titration night for its treatment. ${ }^{12}$

As the demand for expedient diagnosis of suspected OSA has increased and PSG is relatively inconvenient, expensive and inefficient, portable sleep recording devices have been developed as an alternative approach and are now widely used typically unattended and at home. $^{13}$

Portable sleep recording devices which do not record EEG and have a limited number of channels (4-7) are so-called type-III devices. Type-II sleep studies or non-attended home polysomnography (H-PSG) include an EEG and therefore offer a complete sleep evaluation and home-centred care. ${ }^{14}$

A review which analysed six prospective randomised crossover studies comparing H-PSG to in-lab PSG showed that it can be used to rule out OSA in suspected patients, even in the presence of comorbidities in a way which is beneficial to the patient while demonstrating a good technical performance. ${ }^{14}$

The American Academy of Sleep Medicine (AASM) issued new recommendations concerning type-III and type-IV recording devices where portable monitoring is defined to be used as an alternative to attended in-lab PSG for the diagnosis of OSA in patients with a high pretest probability of moderate to severe OSA without comorbidities thereby specially mentioning patients who had a stroke, but also patients with probable nocturnal hypoventilation. The use of out-of-centre sleep testing (OCST) with a limited number of channels has been included in the diagnostic criteria for adult OSA. According to the new International Classification of Sleep Disorders (ICSD) criteria, diagnosis of OSA requires 15 or more predominantly obstructive respiratory events per hour of sleep during a PSG or per hour of monitoring with a portable device (OCST). ${ }^{15}$ But there are also some limitations in the estimation of OSA severity with type-III devices. One concern is the underestimation of SA severity because of limitations in sleep time assessment and the missing scoring of arousals. ${ }^{16}$ Another concern is the night-to-night variability of the apnoea-hypopnoea index (AHI) which can distort results, but is also seen in type-I PSG. ${ }^{17}$ Type-III recordings are often associated with sensor losses and inadequate recordings in $5 \%-30 \%$ of sleep studies. ${ }^{18} 19$
Therefore, attended in-lab PSG is still considered the 'gold standard', but as patients sleep in an artificial (and sometimes unpleasant) laboratory environment, which may result in increased sleep fragmentation, the homerecorded OSA index probably reflects OSA severity more accurately.

\section{SA therapy}

The ability of PAP treatment to lower the risk of serious adverse outcomes after stroke remains controversial because of a substantial risk of bias identified in most studies. ${ }^{6}$ Adherence to PAP therapy is influenced by many factors, including severity of stroke, possible side-effects of PAP therapy, reduction of OSA symptoms, claustrophobia, the patient's perception of disease seriousness, missing family support and costs. Increased air leakage with auto-PAP (APAP) therapy is also associated with reduced adherence ${ }^{20}$ and general management with interventions such as heated humidification, mask optimisation and topical nasal therapy improves adherence. ${ }^{21}$

According to a recent meta-analysis, low mean compliance rates of $37 \%$ in patients who had a stroke are mainly explained by a discomfort with the device, even if patients in very unstable mental and physical conditions are excluded from studies. ${ }^{6}$

\section{CPAP adherence-home versus in-lab titration}

Results of seven randomised trials in otherwise healthy patients with OSA demonstrate equivalent initial continuous positive airway pressure (CPAP) adherence and functional improvement in both the home and the lab titration approach. ${ }^{18}{ }^{22}$ In a recent study by Berry et al, home treatment with APAP and in-lab PSG titration +PAP treatment resulted in equivalent PAP adherence and improvement in sleepiness. ${ }^{23}$

A sleep laboratory in Israel switched from in-lab PSG to home studies in the diagnosis of OSA, which improved test accessibility and reduced waiting times. Patients' satisfaction remained similarly high. The total direct cost of OSA management was reduced, although 597 PAPs were purchased in 2007-2008 as compared with 831 in 2010-2011. ${ }^{18}$

\section{Blood pressure monitoring by means of the pulse transit time (PTT) method}

SA is the most common cause for secondary hypertension, which itself is the most important risk factor for stroke. Thus, it may be interesting to monitor BP before and under PAP therapy. ${ }^{1}$

The antihypertensive effect of PAP has not yet been shown in patients who had a stroke. Therefore, we decided to investigate the nocturnal BP with the PTT method which in contrast to the measurement of BP with 24 hours devices allows a continuous estimation of BP. With this method transient changes can be assessed and related to respiratory events. This method is based on the relation between BP and pulse wave velocity and validation studies revealed a good ability for recognition 
of BP fluctuations. ${ }^{24}$ Thus, in the present study, median and maximum systolic BP before and after PAP therapy will be measured.

\section{Telemedicine and PAP compliance}

Current technology enables PAP devices to monitor pressures applied, air leaks, the AHI and objective adherence and daily send this information to the patient's healthcare provider by means of a modem.

In a study by Fox et al, PAP adherence was improved with the use of a web-based telemedicine system at the initiation of treatment. ${ }^{25}$ After 3 months, mean PAP adherence was significantly higher in the telemedicine arm (191 min per day) than in the standard arm (105 min per day; mean difference $=87 \mathrm{~min}$ ).

AirView is a seamless, securely hosted cloud-based system developed by ResMed for managing patients with sleep-disordered breathing under PAP therapy.
Built-in wireless connectivity enables the sleep physician to seamlessly and securely review therapy data and adjust device settings.

This system allows early detection of problems and appropriate interventions, thereby improving early experience with PAP and potentially improving adherence.

\section{METHODS AND ANALYSIS}

\section{Study design}

A single-blind, monocentre, randomised controlled trial in 55 patients who had a stroke is being conducted at the Neurological Rehabilitation Centre Rosenhügel (Vienna, Austria,) from 18 April 2016 to 18 April 2018. Participants are randomised to either a standard care (SG) or a telemedicine group (TG) (1:1 ratio). Study personnel involved in measurements was blinded (figure 1).

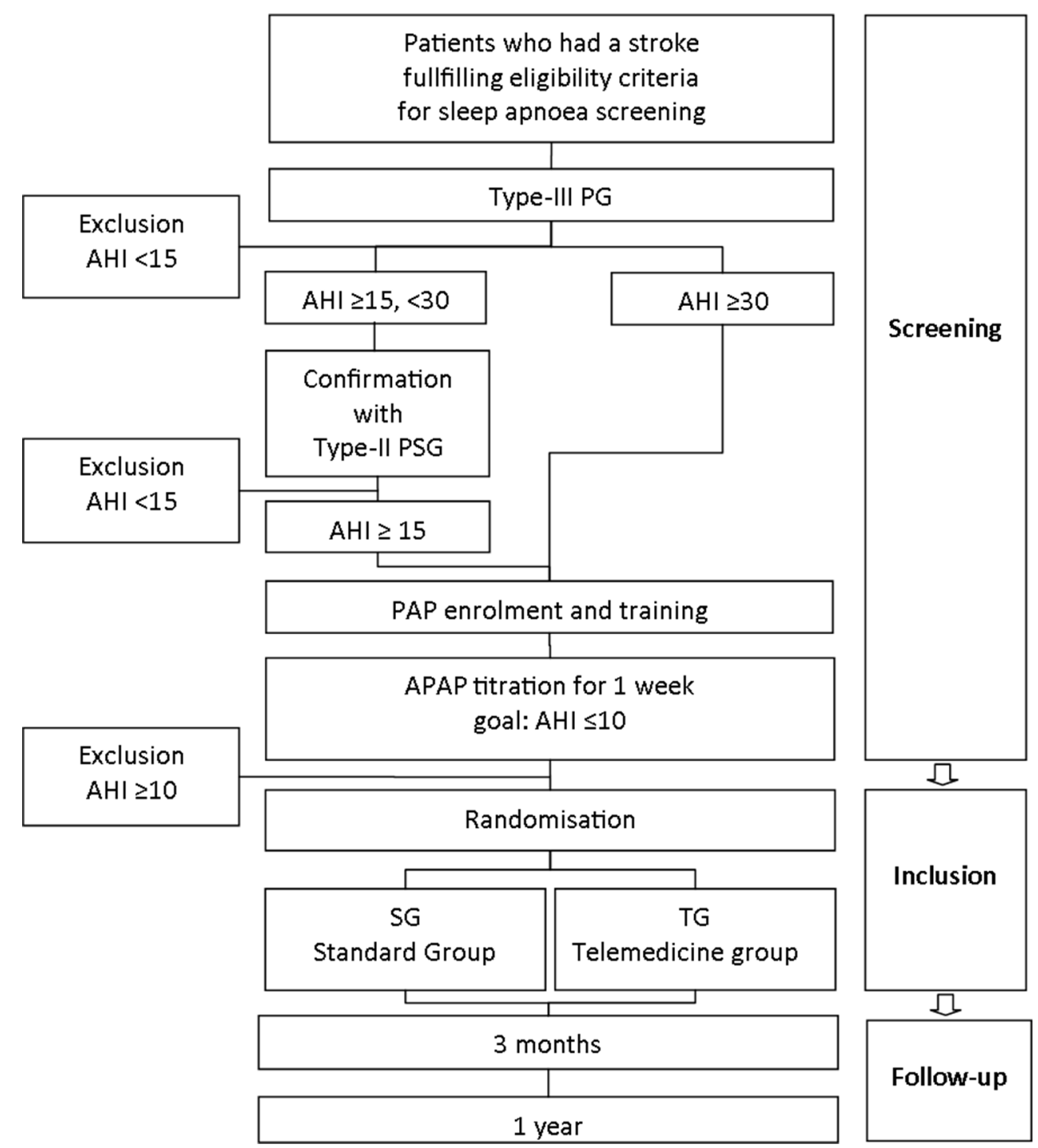

Figure 1 An overview of the study design. AHI, apnoea-hypopnoea index; PAP, positive airway pressure; PSG, polysomnography. 


\section{Primary objective}

The purpose of the present study is to determine whether a telemedical monitoring system is able to improve 3 months PAP adherence in patients who had a stroke with moderate to severe SA, who initially accept CPAP therapy. Consequently, the primary hypothesis is:

H0: Three-month PAP adherence (measured in min of use per day) will be statistically significantly higher in the telemedicine than in the standard treatment group.

\section{Secondary objectives}

Secondary objectives are to:

1. Assess predictive variables for PAP adherence.

2. Compare the feasibility/validity of a non-attended type-III PG with that of a level-II PSG in the diagnosis of SA.

3. Compare the feasibility and validity of an AHI yielded by the PAP device with that obtained during PSG.

4. Assess changes in nocturnal systolic BP due to PAP therapy with the PTT method.

5. Examine the impact of telemonitored PAP therapy on cognitive and motor neurorehabilitation outcome parameters listed at clinical scales (methods) after 3 months and 1 year.

\section{Inclusion and exclusion criteria}

Subacute (>1 month, <1 year post stroke) adult (19-70 years of age) patients who had a stroke referred to the Neurological Rehabilitation Centre Rosenhügel from acute care units of other hospitals or neurologists 'offices are included in the study'. Their diagnosis of a completed stroke has been confirmed by a neurologist based on the history of a sudden onset of a neurological deficit lasting longer than 24 hours, the presence of a neurological deficit on physical examination and a brain lesion compatible with the neurological deficit in CT or MRI of the brain. Patients who accept CPAP therapy after successful titration are asked to take part in the further study process.

Exclusion criteria are as follows: patients with sopor/ coma, patients unable to understand the protocol due to severe cognitive impairments, previously diagnosed SA and established PAP therapy, central SA, chronic obstructive pulmonary disease $>$ Gold III, cancer, chronic kidney disease $>$ stage 4 , coexisting causes of daytime sleepiness (eg, narcolepsy, night or rotating shift-work; self-reported average sleep duration <4hours), a major psychiatric or any other acute medical condition, patients unable or unwilling to comply with the protocol. Physical and neurological examinations are performed in all patients.

\section{Sample size calculation}

To calculate the number of participants needed for an effect size larger than 0.80 , as proposed by Cohen (statistical power analysis for behavioural science), ${ }^{24}$ we based a power calculation on the results of the study by Fox et al in patients with SA. ${ }^{25}$ Therefore, we calculated a two-sided t-test with a significance level of 0.05 and a difference in groups of $114 \mathrm{~min}$ with a SD of $93 \mathrm{~min}$. The result of this calculation was 11 patients in one group. Assuming that $30 \%$ will discontinue PAP treatment at home and further $30 \%$ will reject PAP treatment during inhospital rehabilitation, we aim to include 55 patients in the randomisation process. Based on previously published data, we consider that 168 patients have to be included in the screening process for SA. ${ }^{23}$

\section{Randomisation}

Participants are randomly assigned to the SG or TG, stratified by age (>respectively $<59$ years) with the help of the 'Randomizer for Clinical Trials 1.8.1'. ${ }^{26}$ Randomisation is performed after successful PAP titration and baseline PSG and when the patient has signed the informed consent.

\section{Intervention groups}

Standard group

Patients receive an AirSense 10 AutoSet CPAP. Two days later, they are contacted by their homecare provider and are asked about progress and adherence as well as about any problems that might have occurred.

After 3 months and 1 year of therapy, they are asked to return to the hospital to see their PAP coordinator and physician and discuss any problems with treatment. A type-II PSG is performed at patients' homes to evaluate possible changes in sleep and respiratory variables.

In case of any problems with the therapy, patients are asked to call the homecare provider. A telephone number is attached on the PAP device.

\section{Telemedicine group}

Patients receive the same PAP device as in the SG. In addition, the telemedicine module is activated by the homecare provider. It has been programmed to send physiological information (PAP adherence, PAP pressure applied, mask leakage and residual respiratory events) to a web-based database via a GPRS module every day.

Patients are contacted by their homecare provider after 2 days and are asked about progress and compliance as well as about any problems. The PAP coordinator at the homecare provider reviews the downloaded information every morning except on weekends and holidays and will contact the patient, if any of the events given in table 1 occur.

After 3 months of therapy, patients return to the hospital to see their doctor and the data obtained are reviewed (including PAP pressure, mask leakage, residual respiratory events and compliance). Any problems with treatment may be addressed.

Like in the SG, PSG is performed at patients' homes and clinical scales are completed.

\section{Measurements}

Type-II and type-III sleep studies

PSG and PG are performed by trained staff members using the Somnomedics Diagnostic Systems (SOMNOmedics GmbH, Germany). Patients are studied in their hospital beds. Polygraphic and polysomnographic all-night recordings according to AASM standard criteria 
Table 1 Problems leading to interventions by the homecare provider

$\begin{array}{ll}\text { Event } & \text { Intervention homecare provider } \\ \begin{array}{l}\text { 90th percentile of pressure }>16 \mathrm{~cm} \mathrm{H}_{2} \mathrm{O} \text { or mask leakage } \\ \text { of the } 95 \text { percentiles }>24 \mathrm{~L} / \mathrm{min} \text { or }<4 \text { hour of use for three } \\ \text { consecutive nights }\end{array} & \begin{array}{l}\text { The homecare provider will call the patient or his/her relatives } \\ \text { and will ask about symptoms such as dry mouth, mask issues, } \\ \text { discomfort with the device or any other problems. If the only } \\ \text { issue is low adherence due to a lack of motivation, the homecare } \\ \text { provider will encourage the patient to use PAP. Should there be } \\ \text { any other problems, the homecare provider will discuss the case } \\ \text { with the PAP coordinator (the same person who sees the patients } \\ \text { in the standard arm) and arrange for the patient to talk to or }\end{array} \\ \begin{array}{l}\text { see the PAP coordinator, depending on the patient's responses } \\ \text { and the physiological data obtained. Specific interventions }\end{array} \\ \text { considered standard at the hospital (eg, different mask, chin strap, } \\ \text { modifications of pressure settings, modifications of humidifier } \\ \text { settings, saline nasal sprays) may be performed to improve } \\ \text { compliance. } \\ \text { Contact physician }\end{array}$

AHI, apnoea-hypopnoea index; PAP, positive airway pressure.

are obtained between approximately 20.00 (lights-out) and $6.00 \mathrm{am}$ (lights-on). Time in bed (TIB) is determined by the patients' sleep log.

Polygraphic recordings include oronasal air flow (using thermistor and nasal cannula), thoracic and abdominal effort, oxyhaemoglobin saturation, ECG and determination of the body position. Recordings are used in further investigations if at least 4 hour of data can be obtained. EEG sleep data include six EEG channels (F3-A2, F4-A1, C3-A2, C4-A1, O1-A2, O2-A1) according to the 10/20 system, two electro-oculogram channels (left/right), submental electromyogram (EMG) and tibialis anterior EMG from both legs. All data obtained are scored manually to the criteria developed by the AASM. ${ }^{27}$ Apnoea is scored when there is a drop in the peak signal excursion by $\geq 90 \%$ of pre-event baseline using an oronasal thermal sensor (diagnostic study), PAP device flow (titration study for $\geq 10 \mathrm{~s}$. Hypopnoea is scored when the peak signal excursions drop by $\geq 30 \%$ of pre-event baseline using nasal pressure (diagnostic study), PAP device flow (titration study), for $\geq 10 \mathrm{~s}$ in association with either $\geq 3 \%$ arterial oxygen desaturation. ${ }^{27}$ The diagnosis of a central SA syndrome, as an exclusion criteria requires that more than $50 \%$ of all respiratory events are central. ${ }^{15}$

The AHI is defined as the sum of all apnoeas and hypopnoeas occurring per hour of recording time.

Systolic BP is determined by means of a non-linear algorithm and an individual one-point calibration of the PTT obtained with a cuff-based BP measuring device (SOMNOmedics $\mathrm{GmbH}$, Germany). The number of systolic rises (defined as $>15 \mathrm{~mm} \mathrm{Hg}$ ) in relation to respiratory events and the mean nocturnal systolic BP are determined.

PSG parameters assessed within the study at baseline, 3 months and 1 year:

- $\mathrm{AHI}$

- Desaturation index

- T90 (time $<90 \%$ desaturation)

- Total sleep time (TST) (min)
- TIB (min)

- SE (sleep efficiency, \%)

- S2 (sleep stage 2, \%)

- SWS (slow-wave sleep, \%)

- REM (rapid eye movement sleep, \%)

- Mean systolic BP (mm Hg)

- Number of systolic rises/hour

- Arousal index

- Respiratory- related arousal index

- Periodic leg movement/sleep

- Periodic leg movement-arousal index

- Minimum $\mathrm{SPO}_{2}$

OSA screening

Over the 4-8weeks of neurorehabilitation, each patient enrolled in the study is offered a type-III polygraphic screening for evaluation of OSA (see study flow chart). Within the study period, we expect to screen at least 170 patients.

OSA and referral to PAP training are based on an $\mathrm{AHI} \geq 15$ /hour sleep, as recommended by an international task force on the standardisation of definitions for sleep-related breathing disorders to indicate moderate SA. ${ }^{15}$

If screening with type-III PG reveals an AHI $\geq 15$ and $<30$ / hour, the patient will be offered type-II PSG to confirm the diagnosis of OSA. If type-III PG shows an AHI of $\geq 30$ / hour, the patient will be directly referred to PAP therapy.

Sleep studies $<4$ hour TIB or with less than 4 hours of acceptable signal quality will be repeated if patients agree.

Patients undergoing type-II PSG are asked to complete the Self-Assessment Scale for Sleep and Awakening Quality (SSA). ${ }^{28}$

Patients undergoing type-III PG rate their subjective sleep quality as 'good', 'moderate' or 'bad'.

\section{PAP titration}

All patients referred to PAP therapy receive a 30 min PAP training lesson. A nasal or oronasal mask is fitted to the 
patient's face by the PAP coordinator of our centre, who is a trained respiratory therapist and somnologist certified by the European Sleep Research Society. He has many years of experience with PAP titration.

Patients are provided with an AirSense 10 AutoSet CPAP (Resmed), which includes a humidifier. It is set to auto-titrate at pressures between 6 and $13 \mathrm{~cm} \mathrm{H}_{2} \mathrm{O}$, which reflects our current clinical practice. Pressure limits can be increased up to $16 \mathrm{~cm} \mathrm{H}_{2} \mathrm{O}$, but are decreased to the smallest range that leads to a normalised $\mathrm{AHI}$ in order to avoid overtitration.

Patients accepting PAP therapy after the training session are motivated to use it for at least 4 hours of sleep/ night over 1 week. During the night, patients are coached by trained nurses. Relatives are also trained in using the humidifier and cleaning the mask and the humidifier chamber. The AHI, oximetry and leakage information are collected every day in coaching sessions with the patient. Pressure limits can be increased or decreased to improve patient comfort. If the patient has problems to tolerate high pressures while falling asleep in the first week, the fixed window can be reduced to subtherapeutic pressures (eg, 4-8 mbar) for few nights in order to get used to therapy.

If the Auto CPAP device does not react to obstructive events, titration is too slow or does not decrease (according to PSG or the internal AHI detection), then either a fixed CPAP or a narrow Auto CPAP window will be attached.

If patients consider the interface uncomfortable, it may be changed.

After 1week, patients with a median PAP use of $>4$ hours/night undergo type-II PSG with PAP.

The quality of PAP titration is graded as follows according to Berry et $a 2^{23}$ : optimal (obstructive AHI $\leq 5$ / hour, supine REM sleep on the treatment pressure; desaturation index $\leq 5$ /hour of sleep), good (obstructive AHI $<10 /$ hour, REM sleep on the treatment pressure, desaturation index $\leq 10$ /hour), adequate (AHI $<10$ /hour, supine non-REM (NREM) sleep on the treatment pressure, desaturation index $\leq 10$ /hour). A persisting central $\mathrm{AHI}<5$ is tolerated. If none of these criteria are met, patients will be referred to a local sleep lab and excluded from the study.

\begin{tabular}{lllll}
\hline \multicolumn{4}{l}{ Table 2} & PAP device variables assessed within the study \\
\hline Parameter & Unit & \multicolumn{1}{c}{ Baseline } & 3 months & 1 year \\
\hline Mean AHI & - & $x$ & $x$ & $x$ \\
Mean pressure & $\mathrm{H}_{2} \mathrm{O}$ & $\mathrm{x}$ & $\mathrm{x}$ & $\mathrm{x}$ \\
Mean leakage & $\mathrm{L} / \mathrm{min}$ & $\mathrm{x}$ & $\mathrm{x}$ & $\mathrm{x}$ \\
Days of PAP use & - & & $\mathrm{x}$ & $\mathrm{x}$ \\
$\begin{array}{l}\text { Minutes per day of PAP } \\
\text { use }\end{array}$ & & & $\mathrm{x}$ & $\mathrm{x}$ \\
\hline
\end{tabular}

AHI, apnoea-hypopnoea index; PAP, positive airway pressure.
If patients require a PAP pressure $>16 \mathrm{~cm} \mathrm{H}_{2} \mathrm{O}$ or develop a PAP-emergent central SA, a BiPAP therapy approach will be initiated. See table 2 for variables that will be assessed within the study.

\section{Clinical scales}

The following scores will be obtained from all patients at baseline after 3 months and after 1 year.

- The Epworth Sleepiness Scale (ESS) is a self-administered questionnaire, where respondents are asked to rate their usual chances of dozing off or falling asleep while engaged in eight different activities. ${ }^{29}$ In the present study, the ESS is used to determine subjective sleepiness and its relation to PAP adherence.

- The SSA according to Saletu $e t a l^{28}$ is a self-administered questionnaire with three subcategories, seven questions on sleep quality, eight questions on awakening quality and five questions on physical complaints. ${ }^{28}$ Patients rate the questions on a 4-point scale. Additionally, there are eight questions on bedtime, frequency of nocturnal awakenings, subjective sleep time, and so on. The SSA is used to evaluate subjective sleep quality parameters of home PSG (H-PSG) studies.

- The Quality of Life Index (QoL) according to Mezzich et al consists of 10 questions on health status and quality of life rated on a 10 -point scale. ${ }^{30}$ The mean score is used to investigate QoL in relation to PAP use.

- The PAP Satisfaction Questionnaire comprises three questions on patients' subjective satisfaction with PAP therapy and its influence on symptoms of $\mathrm{SA}^{23}$ It is used in follow-up sessions.

- The National Institutes of Health Stroke Scale is a score calculated from 11 components and is used to quantify the severity of strokes. ${ }^{31}$ The 11 components are level of consciousness, best gaze, visual fields, facial palsy, arm motor, leg motor, limb ataxia, sensory, best language, dysarthria and extinction and inattention. These 11 components are then summed and the score correlates with stroke severity.

- The Timed Up and Go Test is a simple test used to assess a person's mobility. It is based on the time it takes a person to rise from a chair, walk $3 \mathrm{~m}$, turn around, walk back to the chair and sit down. ${ }^{32}$

- The Nine Hole Peg Test is a test to assess motor function. Developed in 1985 by Mathiowetz and colleagues, ${ }^{33}$ it is a valid and reliable test to assess dexterity in individuals with stroke. ${ }^{34}$ Patients have to put nine wooden pegs into the holes of a peg board placed in front of them. Afterwards, they have to take them out again. One practical trial per arm should be conducted prior to timing the test. The time used is noted. ${ }^{35}$

- The Barthel Index was introduced in 1965 and then developed further. ${ }^{36} 37$ It consists of 10 questions (eating, bathing, washing, dressing, bowels, bladder, toilet use, transfers, mobility, stair climbing) and 
ranges from 0 to 100 points. Within the present study, the Heidelberger Criteria are used.

- The Montreal Cognitive Assessment Test (MOCA) is a brief one-page screening tool validated in mild cognitive impairment. ${ }^{38}$ The highest possible score is 30 . People with scores $\geq 26$ are categorised as normal cognitive function. In the present study, the MOCA is used to evaluate the influence of PAP use on this cognitive outcome variable.

- A medical history, with special consideration of stroke risk factors, as well as a list of current medication (eg, antihypertensive, lipid-lowering and antidiabetic drugs, sleep aids, muscle relaxants) are obtained.

\section{Telemedical interventions}

In all interventions based on telemedical data, the following information is recorded:

Number of phone calls because of:

- Reduced PAP use.

- Nose/throat problems.

- Problems with the mask.

- Handling problems of the PAP device.

These data will also be compared with phone calls from non-telemedicine patients.

\section{User statistics}

Data exploration using descriptive statistical analysis and inferential statistics is performed. Baseline data are shown in frequencies or percentages, means and SD and graphics, $95 \%$ CI are calculated for the differences in percentages and medians. In order to test the normal distribution, histograms and box plots will be applied. If data are skewed, non-parametric tests such as Wilcoxon, Mann-Whitney U test and Kendall'stau and Spearman's correlation coefficients are chosen. Otherwise, t-test and Pearson's correlation coefficients are used. To examine categorical baseline characteristics, $\chi^{2}$ tests are used.

The primary outcome is PAP adherence after 3 months (min of use per day). It will be analysed according to the per-protocol-analysis with t-tests for independent samples if normally distributed. If data are skewed, a Wilcoxon test will be used.

Additionally, in order to examine predictive variables for PAP adherence, univariate and multiple linear and logistic regression analyses will be performed, with age, gender, impairments in activities of daily living and cognitive status as independent variables. Additionally, Bland Altman Plots will be created to compare the feasibility and validity of non-attended level-III PG with those of level-II PSG. A Bland Altman Plot will also be created to compare the validity of the AHIs yielded by the PAP device with that obtained during PSG. For assessing changes in nocturnal systolic BP from baseline to 3 months and 1 year later, ANOVA for repeated measurements will be performed. Finally, to examine the impact of telemonitored PAP therapy on cognitive and motor neurorehabilitation outcome parameters, ANOVA for repeated measurements after 3 months and 1 year will be conducted.
The IBM SPSS Statistics for Windows, V.22 software (IBM, Armonk, New York, USA) is used for all statistical analyses. All tests are two-sided and a $\mathrm{P}$ value $<0.05$ is considered statistically significant.

\section{ETHICS AND DISSEMINATION}

The study is performed in accordance with the relevant guidelines of the Declaration of Helsinki, 1964, as amended in Tokyo, 1975; Venice, 1983; Hong Kong, 1989 and Somerset West, 1996. ${ }^{39}$ Written informed consent for all examinations is obtained on patients' admission. The Ethics Board will be informed of all serious adverse events and any unanticipated adverse effects that occur during the study. All study participants will be insured by the 'Zürich Versicherungs Aktiengesellschaft' (policy no. 25571473-2).

Furthermore, the protocol has been registered and will be updated at Clinicaltrials.gov (Identifier: CT02748681). The study methods are in accordance with the SPIRIT guidelines for reporting randomised trials. ${ }^{40}$

Direct access to the source data will be provided for monitoring, audits, Research Ethics Committee (REC)/ Institutional Review Board (IRB) review and regulatory authority inspections during and after the study. All patients will be coded anonymous and only the study team has access to the original data.

The study results will be disseminated by peer-reviewed publications and conference presentations.

\section{DISCUSSION}

\section{Strength and limitations}

A limitation of the study and our diagnostic in-hospital rehabilitation process might be that patients with severe SA in type-III PG will not undergo a confirmation baseline type-II PSG before PAP therapy.

A comparison of type-III versus type-II sleep studies can therefore be only performed in patients with moderate SA.

We were using the new ICSD three definition for SA, which can be based on a type III-sleep study.

In the clinical context, SA cannot be overestimated by type-III PG, if you carefully visually correct the respiratory values and correlate apnoeas with the desaturation index as well.

To our clinical experience, a severe SA in the screening PG remains a severe SA also in PSG. The diagnosis will not change.

We think that patients who had severely affected stroke need a quick access to therapy.

Acknowledgements We are grateful to Elisabeth Grätzhofer for the editorial assistance.

Contributors STK, MTS and BS designed the study. STK, MTS, AS and JS are responsible for the elaboration and realisation of the project. All authors wrote the manuscript and have read and approved the final version of the manuscript.

Funding This project has been funded in whole with funds from the 'NRZ research account' from NRZ Rosenhügel Vienna. 
Competing interests None declared.

Patient consent Obtained.

Ethics approval Ethikkommission der Stadt Wien. Ethics approval has been obtained by the Clinical Research Ethics Board of Vienna (EK-15-231-1115).

Provenance and peer review Not commissioned; externally peer reviewed.

Open Access This is an Open Access article distributed in accordance with the Creative Commons Attribution Non Commercial (CC BY-NC 4.0) license, which permits others to distribute, remix, adapt, build upon this work non-commercially, and license their derivative works on different terms, provided the original work is properly cited and the use is non-commercial. See: http://creativecommons.org/ licenses/by-nc/4.0/

(C) Article author(s) (or their employer(s) unless otherwise stated in the text of the article) 2018. All rights reserved. No commercial use is permitted unless otherwise expressly granted.

\section{REFERENCES}

1. Somers VK, White DP, Amin R, et al. Sleep apnea and cardiovascular disease: an American heart association/american college of cardiology foundation scientific statement from the american heart association council for high blood pressure research professional education committee, council on clinical cardiology, stroke council, and council on cardiovascular nursing. in collaboration with the national heart, lung, and blood institute national center on sleep disorders research (national institutes of health). Circulation 2008;118:1080-111.

2. Mozaffarian D, Benjamin EJ, Go AS, et al. Heart disease and stroke statistics-2015 update: a report from the American heart association. Circulation 2015;131:434-41.

3. Hermann DM, Bassetti CL. Sleep-related breathing and sleep-wake disturbances in ischemic stroke. Neurology 2009;73:1313-22.

4. Saletu M, Nosiska D, Kapfhammer G, et al. Structural and serum surrogate markers of cerebrovascular disease in obstructive sleep apnea (OSA): association of mild OSA with early atherosclerosis. J Neurol 2006;253:746-52.

5. Saletu M, Sauter C, Lalouschek W, et al. Is excessive daytime sleepiness a predictor of carotid atherosclerosis in sleep apnea? Atherosclerosis 2008;196:810-6.

6. Birkbak J, Clark AJ, Rod NH. The effect of sleep disordered breathing on the outcome of stroke and transient ischemic attack: a systematic review. J Clin Sleep Med 2014;10:103-8.

7. Marin JM, Carrizo SJ, Vicente E, et al. Long-term cardiovascular outcomes in men with obstructive sleep apnoea-hypopnoea with or without treatment with continuous positive airway pressure: an observational study. Lancet 2005;365:1046-53.

8. Yaggi HK, Concato J, Kernan WN, et al. Obstructive sleep apnea as a risk factor for stroke and death. N Engl J Med 2005;353:2034-41.

9. Johnson KG, Johnson DC. Frequency of sleep apnea in stroke and TIA patients: a meta-analysis. J Clin Sleep Med 2010;6:131-7.

10. Kotzian ST, Stanek JK, Pinter MM, et al. Subjective evaluation of sleep apnea is not sufficient in stroke rehabilitation. Top Stroke Rehabil 2012;19:45-53.

11. Yan-fang S, Yu-ping W. Sleep-disordered breathing: impact on functional outcome of ischemic stroke patients. Sleep Med 2009;10:717-9.

12. Fietze I, Penzel T, Alonderis A, et al. Management of obstructive sleep apnea in Europe. Sleep Med 2011;12:190-7.

13. Collop NA. Portable monitoring for the diagnosis of obstructive sleep apnea. Curr Opin Pulm Med 2008;14:525-9.

14. Bruyneel M, Ninane V. Unattended home-based polysomnography for sleep disordered breathing: current concepts and perspectives. Sleep Med Rev 2014;18:341-7.

15. Medicine AAoS. International classification of sleep disorders. 3rd edn, 2014.

16. Golpe R, Jiménez A, Carpizo R. Home sleep studies in the assessment of sleep apnea/hypopnea syndrome. Chest 2002;122:1156-61.

17. Bittencourt LR, Suchecki D, Tufik $S$, et al. The variability of the apnoea-hypopnoea index. J Sleep Res 2001;10:245-51.
18. Safadi A, Etzioni T, Fliss D, et al. The effect of the transition to home monitoring for the diagnosis of OSAS on test availability, waiting time, patients' satisfaction, and outcome in a large health provider system. Sleep Disord 2014;2014:1-6.

19. Masa JF, Corral J, Pereira R, et al. Effectiveness of home respiratory polygraphy for the diagnosis of sleep apnoea and hypopnoea syndrome. Thorax 2011;66:567-73.

20. Valentin A, Subramanian S, Quan SF, et al. Air leak is associated with poor adherence to autoPAP therapy. Sleep 2011;34:801-6.

21. Ballard RD, Gay PC, Strollo PJ. Interventions to improve compliance in sleep apnea patients previously non-compliant with continuous positive airway pressure. J Clin Sleep Med 2007;3:706-12.

22. Rosen CL, Auckley D, Benca R, et al. A multisite randomized trial of portable sleep studies and positive airway pressure autotitration versus laboratory-based polysomnography for the diagnosis and treatment of obstructive sleep apnea: The HomePAP study. Sleep 2012;35:757-67.

23. Berry RB, Sriram P. Auto-adjusting positive airway pressure treatment for sleep apnea diagnosed by home sleep testing. J Clin Sleep Med 2014;10:1269-75.

24. Gesche H, Grosskurth D, Küchler G, et al. Continuous blood pressure measurement by using the pulse transit time: comparison to a cuff-based method. Eur J Appl Physiol 2012;112:309-15.

25. Fox N, Hirsch-Allen AJ, Goodfellow E, et al. The impact of a telemedicine monitoring system on positive airway pressure adherence in patients with obstructive sleep apnea: a randomized controlled trial. Sleep 2012;35:477-81.

26. Randomizer for clinical trials 1.8.1. Secondary Randomizer for clinical trials 1.8.1. https://www.meduniwien.ac.at/randomizer/web/login.php

27. Berry RB, Budhiraja R, Gottlieb DJ, et al. Rules for scoring respiratory events in sleep: update of the 2007 AASM Manual for the Scoring of Sleep and Associated Events. Deliberations of the Sleep Apnea Definitions Task Force of the American Academy of Sleep Medicine. $J$ Clin Sleep Med 2012;8:597-619.

28. Saletu B, Wessely P, Grünberger J, et al. Erste klinische Erfahrungen mit einem neuen schlafanstoßenden Benzodiazepin, Cinolazepam, mittels eines Selbstbeurteilungsbogens für Schlaf- und Aufwachqualität (SSA). Neuropsychiatrie 1987;1:169-76.

29. Johns MW. A new method for measuring daytime sleepiness: the Epworth sleepiness scale. Sleep 1991;14:540-5.

30. Mezzich JE, Cohen NL, Ruiperez MA. A quality of life index: brief description and validation. International Congress of the International Federation for Psychiatric Epidemiology. Spain: Santiago de Compostela, 1996.

31. A. H. The NIH stroke scale a window into neurological status, 2011

32. Podsiadlo D, Richardson S. The timed "Up \& Go": a test of basic functional mobility for frail elderly persons. J Am Geriatr Soc 1991;39:142-8.

33. Mathiowetz V, Volland G, Kashman N, et al. Adult norms for the box and block test of manual dexterity. Am J Occup Ther 1985;39:386-91.

34. Croarkin E, Danoff J, Barnes C. Evidence-based rating of upperextremity motor function tests used for people following a stroke. Phys Ther 2004;84:62-74.

35. Olindo S, Signate A, Richech A, et al. Quantitative assessment of hand disability by the Nine-Hole-Peg test (9-HPT) in cervical spondylotic myelopathy. J Neurol Neurosurg Psychiatry 2008;79:965-7.

36. Mahoney FI, Barthel DW. Functional evaluation: the barthel index. Md State Med J 1965;14:61-5.

37. Collin C, Wade DT, Davies S, et al. The Barthel ADL Index: a reliability study. Int Disabil Stud 1988;10:61-3.

38. McLennan SN, Mathias JL, Brennan LC, et al. Validity of the montreal cognitive assessment (MoCA) as a screening test for mild cognitive impairment $(\mathrm{MCl})$ in a cardiovascular population. J Geriatr Psychiatry Neurol 2011;24:33-8.

39. Dale O, Salo M. The Helsinki Declaration, research guidelines and regulations: present and future editorial aspects. Acta Anaesthesiol Scand 1996;40:771-2.

40. Moher D, Hopewell S, Schulz KF, et al. CONSORT 2010 explanation and elaboration: updated guidelines for reporting parallel group randomised trials. Int J Surg 2012;10:28-55. 